\title{
Laguerre Gaussian beam multiplexing through turbulence
}

\author{
Abderrahmen Trichilii, ${ }^{1 *}$, Thandeka Mhlanga ${ }^{2,3}$, Darryl Naidoo², Angela Dudely ${ }^{2}$, Mourad \\ Zghal $^{1}$ and Andrew Forbes ${ }^{2,3}$ \\ ${ }^{1}$ University of Carthage, Engineering School of Communication of Tunis (Sup'Com), \\ GreS'Com Laboratory, Ghazala Technopark, 2083, Ariana, Tunisia \\ ${ }^{2}$ CSIR National Laser Centre, P.O. Box 395, Pretoria 0001, South Africa \\ ${ }^{3}$ School of Physics, University of KwaZulu-Natal, Private Bag X54001, Durban 4000, South \\ Africa
}

\begin{abstract}
We analyze the effect of atmospheric turbulence on the propagation of multiplexed Laguerre Gaussian modes. We present a method to multiplex Laguerre Gaussian modes using digital holograms and decompose the resulting field after encountering a laboratory simulated atmospheric turbulence. The proposed technique makes use of a single spatial light modulator for the generation of superimposed beam and a second spatial light modulator and a CCD camera for the modal decomposition. The obtained results demonstrate how sensitive the Laguerre Gaussian beams are to atmospheric distortions.
\end{abstract}

Keywords: Mode Division Multiplexing, Digital holography, Modal decomposition, turbulence

\section{INTRODUCTION}

Recently a technique, known as mode division multiplexing (MDM), obtained a lot of attention from the scientific community. ${ }^{1}$ MDM offers the possibility to scale the capacity of a single channel transmission system by several orders of magnitude in order to avoid a future anticipated "capacity crunch". ${ }^{2}$ In MDM transmission, each spatial mode can be used as an independent information carrier. MDM have been widely used to improve the optical communication capacity in both fiber and free space. ${ }^{3-5}$ Two key techniques are required for a mode division multiplexed communication: the multiplexed mode generation and the modal decomposition.

In a free space communication, the atmospheric turbulence aberrations cause severe performance degradation on the propagation of optical signal and may be susceptible to modal crosstalk. The atmospheric turbulence is a stochastic process and different models were proposed to describe the random distortions. The effects of the turbulence have been studied extensively in the case of single mode propagation transmission system. ${ }^{6-8}$

In this context we are interested in multiplexing Laguerre Gaussian (LG) modes in free space. In this paper, we report on experimental results of multiplexing and de-multiplexing of Laguerre Gaussian modes using spatial light modulators. Particularly, the multiplexing digital holograms are encoded by complex amplitude modulation. Using a complete modal decomposition technique, we show that we are able to detect the phase and the amplitude of initially excited modes with high fidelity. We also demonstrate the simulation of atmospheric turbulence in the laboratory using a diffractive plate encoded for Kolmogorov turbulence model. We discuss the impact of different turbulence strengths on the purity of the multiplexed modes after de-multiplexing. We consider this work to be helpful in the case of high bit rate optical communication.

The outline of this paper is organized as follows: In section 2, the theory of mode generation and modal decomposition is presented. Section 3, is devoted to the experimental realization of the Laguerre Gaussian mode multiplexing and detection. In Section 4, the main results are discussed with respect to the applied atmospheric distortions to the multiplexed Laguerre Gaussian modes. Finally, section 5 concludes the paper.

\footnotetext{
*abderrahmen.trichili@supcom.tn; phone: +216 71857 000; fax: +216 71856 829; www.supcom.mincom.tn
} 


\section{CONCEPT AND THEORY}

The aim of our work is to generate a superposition of Laguerre Gaussian modes and then perform radial and azimuthal modal decomposition to recover the initially multiplexed modes with and without atmospheric turbulence. The superposition of an optical field with Laguerre Gaussian modes, in polar coordinates, can be written as follows:

$$
U(r, \Phi)=\sum_{p, l} c_{p, l} E_{p, \ell}^{L G}(r, \Phi)
$$

where $\mathrm{r}=(\mathrm{x}, \mathrm{y})$ represents the two dimensional vector position in the source plane and $\Phi$ is the azimuthal angle. $E_{p, \ell}^{L G}$ is the Laguerre Gaussian mode with radial index $p$ and azimuthal index $\ell$ and $c_{p, l}$ is the corresponding modal content.

Let $\rho_{p, l}=c_{p, l}^{2}$ the modal power of mode $E_{p, \ell}^{L G}$. Since the Laguerre Gaussian modes form a complete orthonormal basis, the modal power can be found as follows:

$$
\rho_{p, l}=\frac{1}{2 \pi} \int_{0}^{\infty} \int_{0}^{2 \pi} t_{p, \ell}(r, \Phi) U(r, \Phi) r \mathrm{~d} r \mathrm{~d} \Phi
$$

The physical implementation of the inner product is the modulation of the multiplexed signal at the origin of the Fourier plane with a transmission function denoted as $t_{p, \ell}(r, \Phi)$. The resulting signal can be calculated numerically as follows:

$$
I_{l, p}(0)=\mathcal{F}(U(r, \Phi)) \otimes \mathcal{F}\left(t_{p, \ell}(r, \Phi)\right)
$$

where $\mathcal{F}$ represents the Fourier transform and $\otimes$ denotes the convolution process. Eq. 3 is then expressed as:

$$
I_{l, p}(0)=\frac{\exp (i 2 k f)}{i \lambda f} \int_{0}^{\infty} \int_{0}^{2 \pi} t_{p, \ell}(r, \Phi) U(r, \Phi) r \mathrm{~d} r \mathrm{~d} \Phi
$$

where $f$ in the focal length used in the decomposition $\lambda$ is the wavelength and $k$ is the wavenumber. The transmission function of the modal decomposition is given by:

$$
t_{p, \ell}(r, \Phi)=E_{p, \ell}^{L G}(r, \Phi)
$$

In the presence of atmospheric turbulence aberrations for Kolmogorov turbulence model, the initial LG field $\left(E_{p, \ell}^{L G}\right)_{i}$ becomes:

$$
E_{p, \ell}^{L G}=\left(E_{p, \ell}^{L G}\right)_{i} \exp \left(i \pi b Z_{n}^{m}\right)
$$

where $Z_{n}^{m}$ is the Zernike polynomial of radial order $n$ and azimuthal order $m$, and $b$ is the aberration strength in the form of a simple weighting factor. ${ }^{9}$ The turbulence aberration strength can also be characterized by the Strehl ratio (SR) as explained in Ref. 10.

Now we demonstrate how to experimentally multiplex LG modes and perform radial and azimuthal modal decomposition using spatial light modulators and digital holograms. We insert a turbulence diffractive plate to simulate the atmospheric turbulence.

\section{EXPERIMENTAL REALIZATION}

The experimental setup comprises two parts $\mathrm{P}_{1}$ and $\mathrm{P}_{2}$ : the generation and then decomposition of the multimode field, as shown in Fig. 1. A HeNe laser was expanded through a telescope formed by two lenses $\mathrm{L}_{1}$ and $\mathrm{L}_{2}$ onto the liquid crystal display (LCD) of a spatial light modulator (SLM) (HoloEye, PLUTO-VIS, with $1920 \times 1080$ pixels of pitch $8 \mu \mathrm{m}$ and calibrated for a $2 \pi$ phase shift at $633 \mathrm{~nm}$ ). To execute LG mode multiplexing, we used amplitude modulation using the technique explained in Ref 11. The resulting superposition beam was then directed to a second SLM, denoted as $\mathrm{SLM}_{2}$ for achieving the modal decomposition. This was accomplished by performing an inner product of the incoming optical field with the transmission function set to $E_{p, \ell}^{L G}(r, \Phi)$, for particular $\ell$ and $p$. The azimuthal index $\ell$ are ranging from -10 to 10 and the radial index $p$ are ranging from 0 to 5 . 


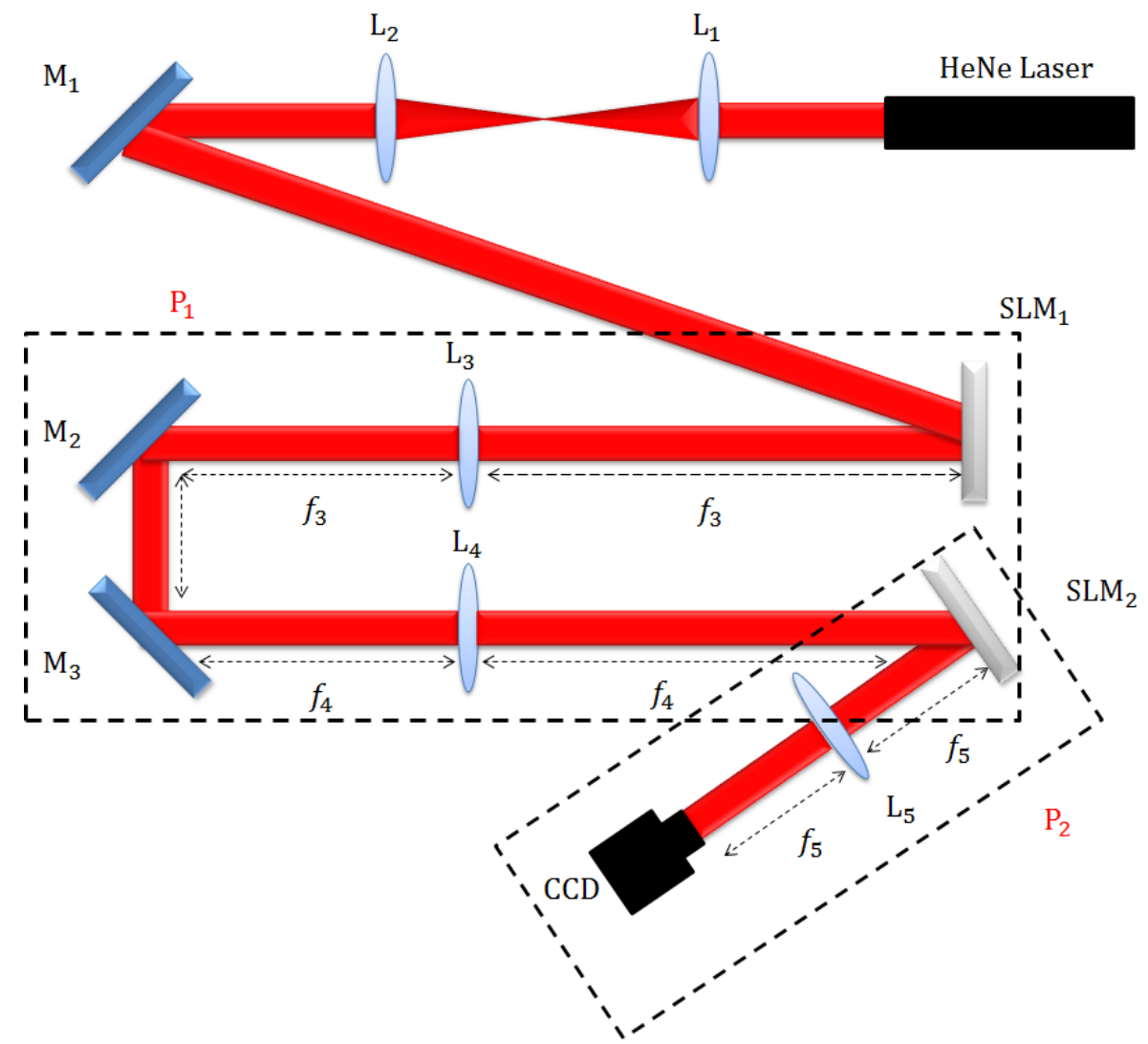

Figure 1. A schematic of the experimental setup for accomplishing the decomposition of a Laguerre Gaussian field. The Lenses $\mathrm{L}_{1}, \mathrm{~L}_{2}, \mathrm{~L}_{3}, \mathrm{~L}_{4}$ and $\mathrm{L}_{5}$ have focal lengths $f_{1}=40 \mathrm{~mm}, f_{2}=750 \mathrm{~mm}, f_{3}=500 \mathrm{~mm}, f_{4}=500 \mathrm{~mm}$ and $f_{5}=150$ $\mathrm{mm}$, respectively, and $\mathrm{SLM}_{1}$ and $\mathrm{SLM}_{2}$ denote two spatial light modulators. $\mathrm{M}_{1}, \mathrm{M}_{2}$ and $\mathrm{M}_{3}$ represent mirrors. The detector is a CCD camera.

Fig. 2(b) and Fig. 2(c) show respectively, experimental and theoretical images of a four multiplexed mode-beam taken at the far field of $\mathrm{SLM}_{1}$ and generated by the digital hologram depicted in Fig. 2(a).

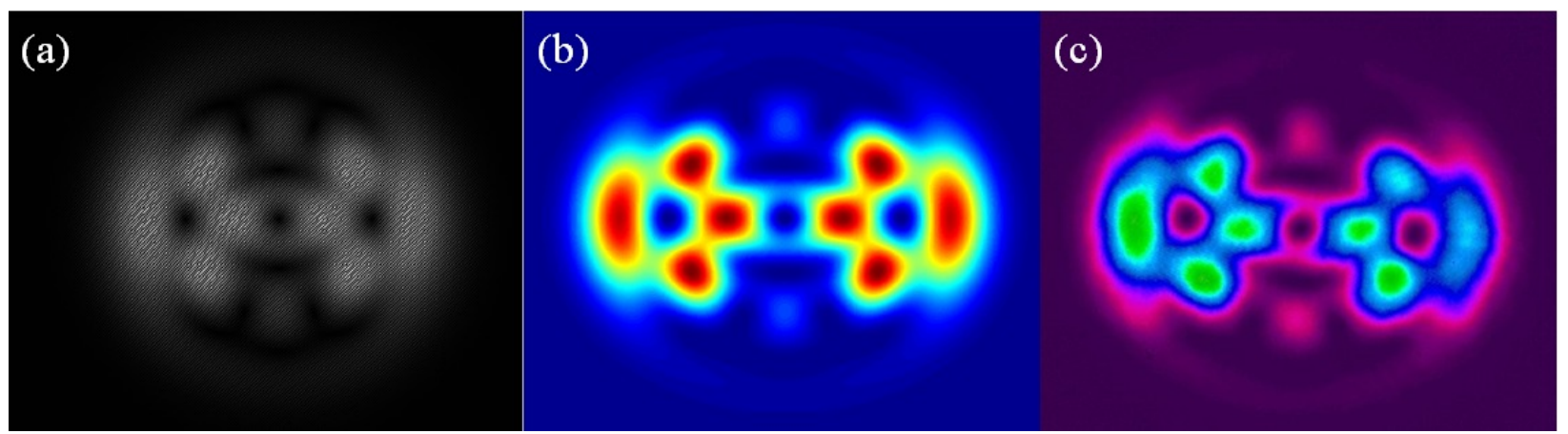

Figure 2. Images (a) of digital hologram (b) theoretical and (c) experimental of four multiplexed LG modes with $\mathrm{p}=1$ and $\ell=1, \ell=3, \ell=5$ and $\ell=7$

\section{EXPERIMENTAL RESULTS AND DISCUSSION}

We first create a superposition of two Laguerre Gaussian modes having opposite azimuthal indexes $\ell=5$ and $\ell=-5$, and same radial index $\mathrm{p}=0$ with equal weighting. The resulting signal is equal to:

$$
U(r, \Phi)=E_{0,-5}^{L G}(r, \Phi)+E_{0,5}^{L G}(r, \Phi)
$$


In this study case, we only apply an azimuthal index decomposition. Five different turbulence strengths [corresponding to $\mathrm{Strehl}$ ratios values of $\mathrm{SR}=0.45, \mathrm{SR}=0.25, \mathrm{SR}=0.10, \mathrm{SR}=0.05$, and $\mathrm{SR}=0.03$, from weak to strong, were used with the impact on the Laguerre Gaussian modes shown in Fig. 3. Without the turbulence plate, the results show a pure azimuthal spectrum with equal modal content for $\ell=5$ and $\ell=-5$ as seen in Fig. 3(a). At weak and moderate turbulence levels ( $\mathrm{SR}=0.45$ and $\mathrm{SR}=0.25$ ), the azimuthal spectrums spread into neighboring modes but $\ell=5$ and $\ell=-5$ still have the higher modal contents [Fig. 3(b), Fig. 3(c)]. As the turbulence becomes stronger the $\ell$ spectrum becomes wider which induces higher crosstalk values.
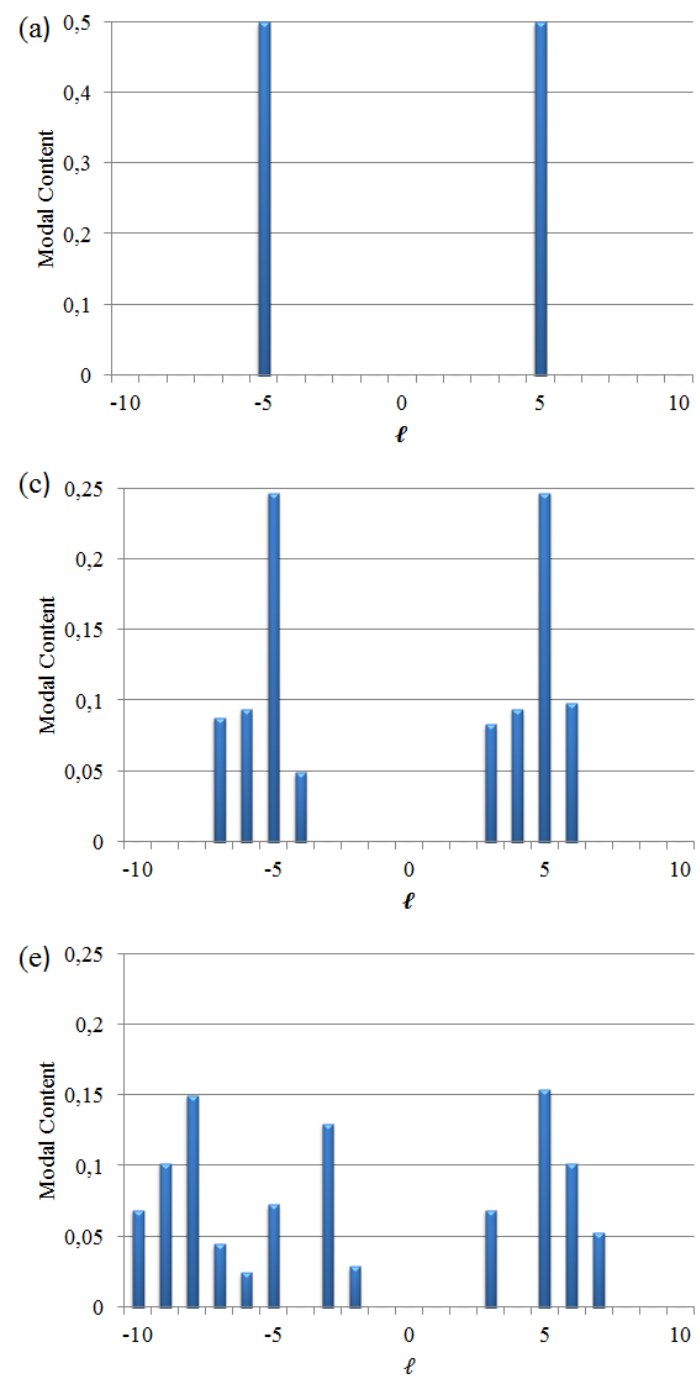
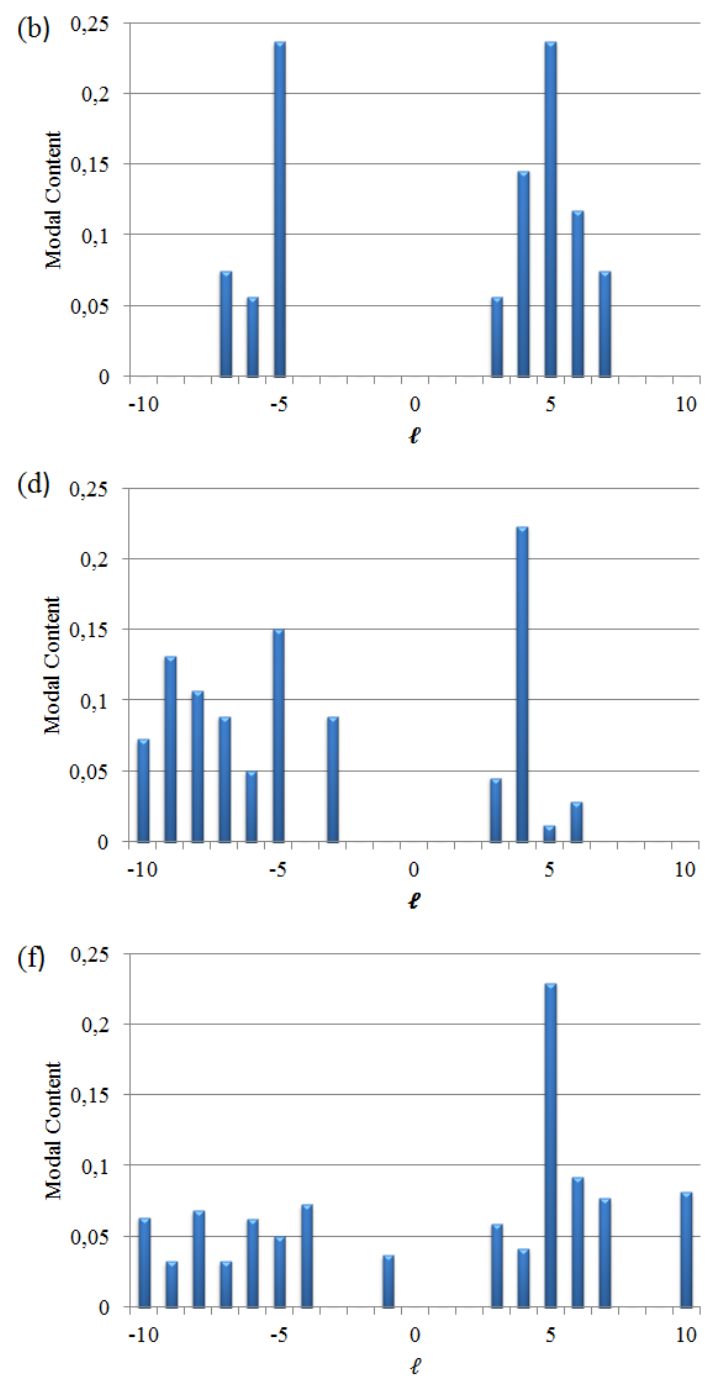

Figure 3. (a) $\ell$ decomposition spectrum without turbulence and (b), (c), (d), (e) and (f) $\ell$ decomposition spectra for $\mathrm{SR}=0.45, \mathrm{SR}=0.25, \mathrm{SR}=0.10, \mathrm{SR}=0.05$, and $\mathrm{SR}=0.03$, respectively

Next we multiplex four LG modes with same radial index $p=1$ and azimuthal indices $\ell=1, \ell=3, \ell=5$ and $\ell=7$. All the modes have the same modal content. The resulting field can be written:

$$
U(r, \Phi)=E_{1,1}^{L G}(r, \Phi)+E_{1,3}^{L G}(r, \Phi)+E_{1,5}^{L G}(r, \Phi)+E_{1,7}^{L G}(r, \Phi)
$$

Without turbulence we obtain a perfect spectrum with no crosstalk on $\ell$ or $p$ as observed in Fig. 4(a). These results show the versatility of our decomposition technique in a perfect medium. At strong turbulence $(\mathrm{SR}=0.10)$, the $p$ spectrum broadens and so $\ell$ spectrum does, as seen in Fig. 4(b). 
(a)

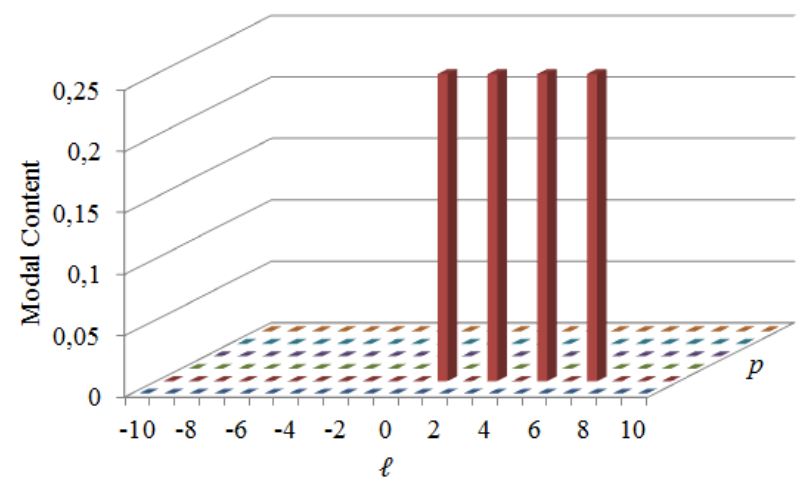

(b)

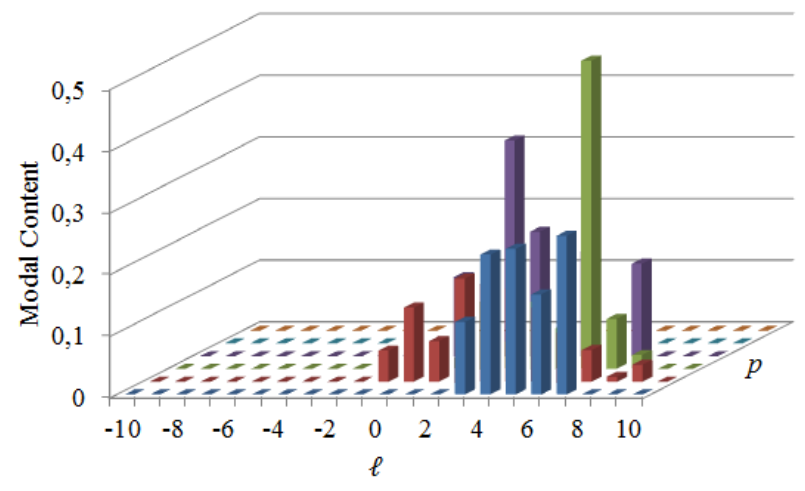

Figure 4. LG radial and azimuthal spectrum for initially generated modes with $\mathrm{p}=1$ and $\ell=1, \ell=3, \ell=5$ and $\ell=7$ (a) without plate and (b) for $\mathrm{SR}=0.1$

The results show the high sensitivity of the LG modes to atmospheric turbulence.

\section{CONCLUSION}

We demonstrate a technique to multiplex and decompose Laguerre Gaussian modes using digital holograms. We also investigate the impact of the atmospheric turbulence on the mode multiplexing. We consider this work to be useful for optical modes transmission where we demonstrate that mode generation and mode decomposition could be highly controlled.

\section{ACKNOWLEDGMENTS}

This work has been supported by African Laser Centre (ALC) project "Towards spatial mode control in fibres for high bit rate optical communication".

\section{REFERENCES}

1. Li, G., and Liu, X., "Focus Issue: Space Multiplexed Optical Transmission," Optics Express 19(17), 1657416575 (2011).

2. Chralyvy, A., "The Coming Capacity Crunch," in [35th European Conference on Optical Communication, 2009. ECOC'09], Plenary paper (2009).

3. Sleiffer, V. A. J. M., Jung, Y., Veljanovski, V., Van Uden, R. G. H., Kuschnerov, M., Chen, H., Inan, B., Grüner Nielsen, L., Sun, Y., Richardson, D. J., Alam, S. U., Poletti, F., Sahu, J. K., Dhar, A., Koonen, A. M. J., Corbett, B., Winfield, R. , Ellis, A. D. , and De Waardt, H., "73.7 Tb/s (96 x 3 x 256-Gb/s) ModeDivision-Multiplexed DP-16QAM Transmission with Inline MM-EDFA," Optics Express 20, B428-B438 (2012).

4. Wang, J., Yang, J. Y., M. Fazal, Irfan, Ahmed, N, Yan, Y., Huang, H, Ren, Y, Yue, Y, Dolinar, S, Tur, M., and E. Willner, Alan, "Terabit free-space data transmission employing orbital angular momentum multiplexing," Nature Photonics 6, 488496 (2012).

5. Huang, H., Xie, G., Yan, Y., Ahmed, N., Ren, Y., Yue, Y., Rogawski, D., Tur, M., Erkmen, B., Birnbaum,K., Dolinar, S., Lavery, M. Padgett, M., and Willner, A. E., "100 Tbit/s Free-Space Data Link using Orbital Angular Momentum Mode Division Multiplexing Combined with Wavelength Division Multiplexing," in [Optical Fiber Communication Conference/National Fiber Optic Engineers Conference 2013], (2013).

6. Ricklin, J. C. and Davidson, F. M., "Atmospheric turbulence effects on a partially coherent Gaussian beam: Implications for free-space laser communication ," J. Opt. Soc. Am. A, 19, 1794-1802 (2002). 
7. Malik, M., O'Sullivan, Rodenburg, M. B., Mirhosseini, M., Leach, J., Lavery, M. P. J., Padgett, M. J., and Boyd, R. W., "Influence of atmospheric turbulence on optical communications using orbital angular momentum for encoding," Optics Express 20, 13195-13200 (2012)

8. Jiang, Y., Wang, S., Zhang, J., Ou, J., and Tang, H., "Spiral spectrum of Laguerre-Gaussian beam propagation in non-Kolmogorov turbulence," Optics Communications 303, 38-41 (2013).

9. Born, M., and Wolf, E., [Principles of Optics], Cambridge University, 7th ed. (1991).

10. Janssen, A., van Haver, S., Dirksen, P., and Braat, J., "Zernike representation and strehl ratio of optical systems with numerical aperture," J. Mod. Opt. 55(7), 1127-1157 (2008).

11. Arrizon, V., Carrada, I. R. R., and Conzalez, L. A., "Pixelated phase computer holograms for the accurate encoding of scalar complex fields," J. Opt. Soc. Am. A 24, 3500-3507 (2007). 